\title{
EFECTO DE INOCULACIÓN MICORRÍZICA ARBUSCULAR COMBINADA CON FERTILIZACIÓN NITROGENADA EN EL PASTO SABOYA (Megathyrsus maximus)
}

\section{EFFECT OF ARBUSCULAR MYCORRHIZAL INOCULATION COMBINED WITH NITROGEN FERTILIZATION ON SAVOY GRASS (Megathyrsus maximus)}

\author{
Jimmy Ivan Zambrano Velasquez ${ }^{1}$, Manuel de Jesús Peña ${ }^{2}$, Hilder Enrique Vera Saldarriaga ${ }^{1}$ \\ ${ }^{1}$ Universidad Técnica de Manabí, Avenida Urbina y Che Guevara Km 21ำ vía Chone-Boyacá \\ ${ }^{2}$ Escuela Superior Politécnica Agropecuaria de Manabí Manuel Félix López, ubicada en el km 2.7 vía Calceta-E1 Morro-E1 Limón
}

Email: jzambrano1657@utm.edu.ec

\section{Información del artículo}

Tipo de artículo:

Artículo original

Recibido:

03/02/2021

Aceptado:

$15 / 06 / 2021$

Licencia:

CC BY-NC-SA 4.0

Revista

ESPAMCIENCIA

12(1):46-55

DOI:

https://doi.org/10.51

260/revista_espamci

encia.v12i1.239

\section{Resumen}

La presente investigación es una revisión bibliográfica que tiene por objetivo examinar el efecto de la inoculación micorrízica arbuscular combinada con la fertilización nitrogenada sobre el pasto Saboya (Megathyrsus maximus). Para sustentar teóricamente este estudio se analizaron 80 artículos obtenidos mediante Google Académico a través de fuentes primarias como investigaciones originales, tesis de grado, de maestría; y de fuentes secundarias extraídas de bases de datos como Dialnet, Redalyc y Scielo, asimismo para la búsqueda bibliográfica se determinó palabras clave pasto Saboya (Megathyrsus maximus), fertilización nitrogenada, inoculación micorrízica arbuscular y uso combinado de fertilización nitrogenada e inoculación micorrízica arbuscular. Se concluyó que la inoculación de hongos micorrízicos arbusculares conjuntamente con la fertilización nitrogenada sobre el pasto Saboya constituye una opción válida para optimizar la absorción de minerales y nutrientes de este género mejorando su rendimiento y calidad, lo cual favorece el perfil alimentario del ganado en el cantón Chone y el país. Se evidenció que, en este sentido, el desafío más importante para el Ecuador es fomentar líneas investigativas dirigidas a implementar ambos métodos de fertilización para alcanzar elevados índices de productividad del pasto Saboya, tomando en cuenta factores como el tipo de suelo, la selección de cepas eficientes y el manejo de cantidades de fertilizante nitrogenado acordes a las necesidades reales del suelo.

Palabras clave: Inoculación micorrízica arbuscular, fertilización nitrogenada, rendimiento del pasto Saboya

\begin{abstract}
The present research is a bibliographic review that aims to examine the effect of arbuscular mycorrhizal inoculation combined with nitrogen fertilization on Savoy grass (Megathyrsus maximus). To theoretically support this study, 80 articles obtained through academic google with primary sources such as original research, graduate thesis, master's thesis; and from secondary sources extracted from databases such as Dialnet, Redalyc and Scielo, also for the bibliographic search Savoy grass (Megathyrsus maximus), nitrogen fertilization, arbuscular mycorrhizal inoculation and combined use of nitrogen fertilization and arbuscular mycorrhizal inoculation were determined as keywords. It was concluded that the inoculation of arbuscular mycorrhizal fungi together with nitrogen fertilization on Savoy grass constitutes a valid option to optimize the absorption of minerals and nutrients of this genus, improving their performance and quality, which favors the feeding profile of cattle in Chone canton and the country. It was evidenced that, in this sense, the most important challenge for Ecuador is to promote research lines aimed at implementing both fertilization methods to achieve high productivity rates of the Savoy grass, taking into account factors such as the type of soil, the selection of efficient strains and the management of nitrogen fertilizer amounts according to the real needs of the soil.
\end{abstract}

Keywords: Arbuscular mycorrhizal inoculation, nitrogen fertilization, Savoy grass yield 


\section{INTRODUCCIÓN}

En la actualidad constituye una preocupación del ámbito ganadero encontrar las vías más eficaces para incrementar la producción de pastos y forrajes, pues esto se traduce en aumento de la producción animal. Sin embargo, aún son insuficientes las investigaciones científicas para alcanzar los niveles de producción de pastos a que se aspira en la región Latinoamericana y especialmente en Ecuador.

De acuerdo a estudios investigativos desarrollados en la región entre las principales causas que afectan desfavorablemente la producción ganadera es la baja fertilidad de los suelos como consecuencia de la poca disponibilidad de nitrógeno, componente mineral con mayor impacto sobre la producción de pasto y forraje en los suelos tropicales (Santos et al., 2002; Bernal, 2003).

El nitrógeno es considerado un nutriente de importancia gracias a que favorece el aumento del tamaño de la hoja en las plantas, el diámetro de las raíces, el desarrollo de nuevos brotes, la absorción de otros nutrientes, la digestibilidad, la producción de materia seca así como el incremento del contenido de proteína cruda en pastos y forrajes (Cabalceta, 1999; León, 2003; Castro et al., 2009; González, 2015).

De acuerdo con el planteamiento anterior la aplicación de fertilización nitrogenada fortalece la producción ganadera al incrementar la oferta y calidad del pasto, por lo cual constituye una estrategia acertada siempre que se tome en cuenta las necesidades del suelo tratado (Cáceres et al., 1989; Mendoza, 2002; Pérez, 2014; López, 2015).

No obstante, la fertilización a base de este componente mineral tiene una efectividad generalmente inferior al $50 \%$, debido a las pérdidas del nitrógeno aplicado por lo cual ocurre un incremento de los costos ambientales, (Blaxter et al., 1971; Reynolds, 1982; Peoples et al., 1994; Whitehead, 1995; González, 2015).

Si bien la productividad de los pastos depende del nitrógeno para conseguir cosechas satisfactorias (Castro et al., 2009), constituye una prioridad contar con mecanismos sustentables para fertilizar los suelos y preservar el medio ambiente.

En este sentido, la producción de pastos se ha orientado hacia la utilización de fuentes renovables donde el empleo del potencial de fijación biológica del nitrógeno cobra vital importancia para complementar o reemplazar la fertilización nitrogenada (Alarcón y Ferrera-Cerrato, 2000; Bernal y Moralez, 2006; Cárdenas et al., 2014; Pérez, 2014; Aguilar et al., 2015).

Una de las alternativas de mayor aceptación para complementar la fertilización mineral es el manejo de la simbiosis micorrízica, pues contribuye a ampliar el área radicular de la planta posibilitando una mejor absorción de los nutrientes del suelo (Barrera, 2009; Cano, 2011; González et al., 2015).

La inoculación de micorrizas favorece la productividad de los cultivos, la regeneración de comunidades vegetales degradadas el equilibrio del ecosistema y el desarrollo de sistemas agrícolas más eficientes (Riera y Medina, 2005; Cardona et al., 2008; Guerra, 2008; Noda, 2009; Cárdenas et al., 2014; Seguel, 2014; González et al., 2015).

Dentro de la gama de micorrizas, los hongos micorrízicos arbusculares han tenido mayor aceptación en los sistemas ganaderos debido a que mediante sus interacciones con otros microorganismos del suelo a través de las raíces y la rizósfera, permiten aumentar la fijación del nitrógeno (Cuenca et al., 2007; Castro, 2009; Pérez et al., 2011).

Varios autores señalan entre los beneficios de la micorriza arbuscular su capacidad para estimular la absorción de fósforo y otros nutrientes, su efectividad para impulsar funciones como la remoción de toxinas, la solubilización de fosfatos orgánicos e inorgánicos, así como la mejora de la estructura del suelo, de la resistencia a las plagas y a la sequía (Maldonado, 2008; Jiménez, 2009; Montaño et al., 2009; Parodi y Pezzani, 2011; Lozano et al., 2015; Garzón, 2016; Ramirez et al., 2017).

La inoculación de hongos arbusculares en los cultivos de pastos constituye una opción válida para disminuir la cantidad de fertilizantes utilizados, así como reducir el tiempo de entrada de animales a pastoreo y el tiempo de establecimiento de árboles para los sistemas silvopastoriles, lo cual permite un sistema de producción más rápido, limpio y eficiente (Blanco y Salas, 1997; Bolívar et al., 2009; Molina et al., 2005).

En este sentido, algunos estudios también aportan evidencias sobre la conveniencia del uso de micorrizas como método para elevar la productividad en las gramíneas, y como parte de estas, del pasto Saboya (Megathyrsus maximus) (Ramírez et al., 2017).

Varios autores indican que el pasto Saboya, también conocido como guinea, chilena o cauca, es gramínea de origen africano que se adapta fácilmente a diversas condiciones climatológicas y se caracteriza por su resistencia al pisoteo del ganado durante el pastoreo, a la sequía extensa, por su alto valor nutritivo y su adecuado nivel de respuesta ante los diferentes sistemas de manejo (Álvarez et al 2016a; Álvarez et al 2016b; Cunalata, 2019; Morán, 2019; Ramírez et al, 2012; Verdecia et al, 2008). En este sentido, una de las zonas cuyas extensiones de pastizales de aproximadamente $163 \quad 441,70$ hectáreas poseen un alto predominio de pasto Saboya es el cantón Chone, siendo considerado el principal abastecedor de 
ganado en Ecuador (Memoria Técnica Cantón Chone, 2013), datos que ratifican la importancia de fomentar su rendimiento y producción.

Sin embargo, se ha señalado que la falta de investigaciones para promover el incremento del rendimiento del pasto Saboya afecta considerablemente las posibilidades de expansión y los índices de productividad del mismo (Macías et al., 2017).

Si bien el pasto Saboya asimila favorablemente la fertilización nitrogenada (Segura, 2007; França et al., 2007; Mesquita y Neres, 2008; Vélez, 2009; Cerdas y Vallejos, 2011; Ceballos, 2019) los costos económicos y ambientales de su uso se pueden aminorar si se complementa con la inoculación micorrízica, lo cual resulta de gran interés teniendo en cuenta las grandes extensiones de cultivos de este género en el territorio ecuatoriano.

En la literatura se hallan estudios acerca del efecto de la inoculación micorrízica arbuscular conjuntamente con la fertilización nitrogenada sobre el pasto Saboya, en los cuales se ha demostrado el incremento del rendimiento y la calidad de este género (Calderón y González, 2007; Ramírez et al., 2017; Rosales et al., 2017a).

Sin embargo, en Ecuador este tipo de investigaciones aún son escasos, por lo que se carece de información acerca del efecto de ambos métodos de fertilización en el pasto Saboya, ello evidencia la necesidad de implementar estudios que avalen su efectividad dada la importancia de este tipo de pasto para la producción ganadera en el territorio.

Por tal motivo el presente trabajo de revisión tiene como objetivo examinar el efecto de la inoculación micorrízica arbuscular combinada con la fertilización nitrogenada sobre el pasto Saboya.

\section{MATERIALES Y MÉTODOS}

Los artículos de revisión contribuyen a situar y sustentar teórica y conceptualmente los aportes científicos existentes gracias a la constante evolución en determinada temática de interés, permite localizar los resultados más relevantes actuales y pasados, sirve como guía metodológica para las investigaciones a la vez que permite identificar las limitaciones y carencias teóricas o metodológicas existentes respecto al tema abordado (Arnau y Sala, 2020; Guirao-Goris et al., 2008).

Al ser el presente un estudio de revisión, en este apartado se describirán los métodos empleados para su redacción, tomando en cuenta aspectos como la estrategia de búsqueda de la literatura científica consultada, los criterios de selección, el período en que se realizó y las latitudes donde fueron realizadas dichas investigaciones.
A su vez, se consideró el número de estudios que sustentan teóricamente el presente trabajo, a partir de criterios de inclusión tales como la relevancia de su aporte al tema analizado y el período de tiempo en que estos fueron realizados.

\section{La estrategia de búsqueda bibliográfica}

El sustento teórico del presente artículo de revisión se obtuvo mediante Google Académico a través de fuentes primarias: investigaciones originales, tesis de grado, de maestría; y de fuentes secundarias extraídas de bases de datos como Dialnet, Redalyc y Scielo.

Para la búsqueda bibliográfica se determinaron a partir de las variables del tema de investigación las palabras clave, siendo seleccionadas pasto Saboya (Megathyrsus maximus), fertilización nitrogenada, inoculación micorrízica arbuscular y uso combinado de fertilización nitrogenada e inoculación micorrízica arbuscular.

La búsqueda se realizó durante el período septiembre 2020abril 2021, se descargaron un total de 136 trabajos entre tesis y artículos científicos, de ellos se analizaron en profundidad y de manera crítica 80 de ellos por ser más afines con el problema de investigación.

Los estudios analizados son fundamentalmente de América Latina (Brasil, Chile, Colombia, Costa Rica, Cuba) y Estados Unidos, abarcan un período amplio, desde la década de los 90 hasta la actualidad y algunos clásicos que datan de años anteriores, los cuales se tomaron en cuenta por su aporte relevante para la presente investigación.

\section{Criterios de selección de los artículos}

Los criterios de selección se determinaron de acuerdo al objetivo del trabajo: examinar el efecto de la inoculación micorrízica arbuscular combinada con la fertilización nitrogenada sobre el pasto Saboya.

Para ello se tomaron en cuenta aspectos como título, los autores, el resumen, los resultados y las conclusiones, con el propósito de determinar si el trabajo aporta información útil sobre el tema investigado, la credibilidad y experticia de los autores examinados, el cumplimiento de criterios de calidad metodológica, el rigor científico de los artículos y tesis consultados, así como el nivel de aplicación de sus resultados obtenidos en el contexto objeto de estudio.

\section{RESULTADOS Y DISCUSIÓN}

En el siguiente apartado, se exponen los principales aportes de las investigaciones analizadas en relación con el pasto Saboya, sus características, los métodos de producción y su nivel de rendimiento. 
Asimismo, se examinan los resultados más relevantes acerca de su productividad bajo condiciones de fertilización nitrogenada, fertilización por medio de la inoculación de micorrizas arbusculares y respecto al uso combinado de ambos métodos de fertilización.

Se presentan las consideraciones respecto a las potencialidades del uso combinado de ambos métodos de fertilización en los cultivos de pasto Saboya en Ecuador.

\section{Producción, rendimiento y métodos de plantación del pasto Saboya}

El pasto Saboya Megathyrsus maximus, anteriormente conocida como Panicum maximum, es una gramínea de que produce entre 10 y 30 toneladas de materia seca por hectárea en un año; entre un 10-14\% de proteínas y una digestibilidad de $60-70 \%$ (Álvarez et al., 2016b; Astudillo, 2014; Morán, 2019; Peñaherrera, 2015).

Varios autores plantean que esta especie puede adaptarse a condiciones extremas pues al ser una gramínea de mucha rusticidad soporta el pastoreo extensivo. Sin embargo, no mantiene un equilibrio de producción durante todo el año, tal desequilibrio está relacionado con el factor climático de mayor variabilidad en el trópico que es la precipitación (Álvarez et al., 2016a; Vargas et al., 2014).

Un estudio realizado en Ecuador propone dos métodos para la plantación y expansión del pasto Saboya: por medio de cariopsis o grano empleando 9 a $18 \mathrm{~kg} / \mathrm{ha}$, y a través del material vegetativo, utilizando 12 a $15 \mathrm{~m} 3$ de cepas por hectárea (Loayza, 2008).

\section{El nitrógeno y su uso en la fertilización del pasto Saboya}

Investigaciones realizadas en Colombia, señalan que el nitrógeno forma parte de las proteínas, clorofilas, alcaloides, enzimas de los pastos, los cuales son componentes responsables de regular el crecimiento y formación del material vegetal de dichas gramíneas (INPOFOS, 2003).

Varios estudios han constatado que especies Megathyrsus máximum, extraen 79,0 y 63,0 kg/ha/año de nitrógeno (Bernal, 2003; Verdecia et al., 2008; Nuñez et al., 2019).

También se ha comprobado que la fertilización nitrogenada contribuye al incremento del valor nutritivo producido por las gramíneas forrajeras (Moran, 2002; Pérez, 2014; González, 2015).

Otros estudios señalan que la incorporación de nitrógeno en las gramíneas forrajeras es del 60\%, y el restante $40 \%$ se pierde ya sea por escurrimiento, lavado, desnitrificación, volatilización, inmovilización biológica u otros procesos ocurridos en el suelo, razón por la cual se recomienda que su aplicación mediante la fertilización sea en forma fraccionada para mayor eficiencia (León, 2003).

Por su parte, algunos investigadores señalan que la efectividad de la fertilización nitrogenada aumenta con la luz, la temperatura y la humedad del suelo, por lo cual recomiendan que la dosis total anual de fertilización de nitrógeno sea fraccionada mínimo 3 veces para obtener resultados eficientes, además proponen que su aplicación se realice durante épocas lluviosas o bajo riego, tomando en consideración el tipo de suelo, el período de corte y el nivel de pastoreo (Gutiérrez, 1996; Cabalceta, 1999).

\section{Fertilización a base de micorrizas arbusculares}

Varios estudiosos de las micorrizas señalan las metodologías empleadas para la fertilización con dichos hongos, entre las que destacan por su efectividad: el aislamiento de esporas a partir de suelo, la tinción diferencial de raíces para evidenciar la colonización, metodologías moleculares para la identificación y taxonomía así como la inoculación con esporas, raíces o suelo (Abbott, 1994; Corredor et al., 2003; Aguilar et al., 2015; Valencia y Zúñiga, 2015).

A su vez otro estudio indica que para una evaluación eficaz del aporte de las micorrizas en la fertilización resulta imprescindible realizar un muestreo representativo, en el cual la muestra debe tomarse de los diferentes puntos de muestreo seleccionados a una profundidad entre 0 y $20 \mathrm{~cm}$ en forma de $\mathrm{X}$ o W, para luego de ser homogeneizada $\mathrm{y}$ secada, extraer entre 10 y 100 gr para el posterior aislamiento y análisis de las esporas mediante el tamizaje y centrifugación en gradiente de sacarosa (Corredor et al., 2003).

Igualmente, para el proceso de inoculación los investigadores recomiendan aplicaciones de entre $100 \mathrm{y}$ 150 gr por árbol con una densidad de 50 esporas/g, para un promedio de 107 esporas por planta (Molina et al., 2005).

Por otra parte, el nivel de efectividad de la micorriza aplicada se evalúa a partir de cuantificar la colonización del hongo micorrícico en las raíces y determinar las características del mismo utilizando un estereoscopio (40x) a través del método de intercepto de líneas cuadráticas de las raíces, luego de ser lavadas, acidificadas y tinturadas (Habte y Osorio, 2001; Valencia y Zúñiga, 2015).

Estudio realizado en Colombia señala que las investigaciones sobre el efecto de las micorrizas en especies forrajeras tropicales se han orientado principalmente a las 
leguminosas, en menor proporción a las gramíneas, y entre estas últimas más a las de pastoreo que a las de corte (Barrera, 2015).

De la misma manera, trabajos que datan de la década de los noventa, señalan que las gramíneas responden a la inoculación con micorrizas en suelos con niveles de poca y mediana fertilidad (Evan y Miller 1990).

Algunos autores señalan que estudios realizados sobre la dependencia de micorrizas de diversas especies forrajeras tropicales, incluidas $B$. decumbens, B. brizantha, $B$. humidicola y Panicum maximum actualmente denominada Megathyrsus maximus, revelan que la dependencia micorrízica de estas especies es mayor cuando crecen en suelos de baja fertilidad (González, 2015; Núñez, 2018).

Por otra parte, Barrera señala que la institución Agrotecnia Ltda. de Colombia en investigaciones realizadas durante el período 1997-2000, demostraron que las aplicaciones de micorrizas que oscilaban entre 500 y $800 \mathrm{Kg} / \mathrm{ha}$, generaron una mayor producción, un aumento en la absorción de nutrientes y una mayor tolerancia al ataque de plagas con respecto a aquellas donde no se aplicaron, a su vez detectaron que las mayores producciones de gramíneas se obtuvieron al aplicar mezclas de micorriza - materia orgánica y fertilizante químico obteniéndose producciones superiores en un 25\% (Barrera, 2015).

Si bien existen numerosos trabajos acerca del uso de hongos micorrízicos arbusculares como biofertilizantes, aún falta profundizar el estudio sobre los efectos de la fertilización mineral en ellos, sin embargo se conoce que estos dependen de factores como el tipo de fertilizante, las características del suelo, de los cultivos y del agroecosistema así como del tipo de hongos micorrízicos arbusculares presentes en el suelo (Sieverding, 1991; Blanco y Salas, 1997; Louis y Lim, 1988; Usuga et al., 2008).

En este sentido, investigadores norteamericanos en estudios que datan de los años 90, descubrieron que determinadas especies de micorrizas arbusculares varían en su respuesta a la fertilización y que altos niveles de nitrógeno y fósforo afectan negativamente el funcionamiento de la micorriza mientras que bajas fertilizaciones con potasio son benéficas (Kurle y Pfleger 1994; González et al., 2015).

Igualmente, en un artículo de revisión varios investigadores puertorriqueños indican que la utilización de plaguicidas y fertilizantes sintéticos aumentan la cantidad de fósforo en el suelo y reduce el efecto positivo de las micorrizas, concluyendo que las probabilidades de disminución de los efectos negativos dependen del tipo y cantidades de fertilizante, así como de la especie de micorrizas empleada (Canchani et al., 2018).
Lo que esulta vital considerar estos aspectos en el momento de selección de especies de micorrizas, para evitar que su efectividad sea afectada por su alta sensibilidad al tipo de fertilizante.

\section{Uso combinado de inoculación micorrízica y fertilización nitrogenada sobre el pasto Saboya}

Varios trabajos han demostrado que la inoculación de especies eficientes de HMA constituye una vía efectiva para disminuir las dosis de fertilizantes a aplicar a los pastos, sin reducir su rendimiento ni valor nutricional (Carneiro et al., 2011; González, 2015; González et al., 2015).

De acuerdo con un estudio desarrollado en Cuba se comprueba que determinados tipos de hongos micorrízicos (F. mosseae) poseen una mayor efectividad para colonizar las raíces del pasto respecto a los hongos micorrízicos arbusculares residentes; así mismo demostraron la necesidad de que conjuntamente con su inoculación se adicionen determinadas cantidades de nutrientes, procedentes de fuentes orgánicas o minerales, para alcanzar una mayor ocupación radical y prolongar su efecto en el tiempo (Ramírez et al., 2017).

Dicho estudio con sus resultados también confirma la tesis de que cuando se incluye una cepa eficiente de hongos micorrízicos arbusculares, la cantidad de nutrientes a aplicar para alcanzar un determinado rendimiento suele ser menor que la necesaria para lograr ese mismo resultado en ausencia de inoculación micorrízica, aspecto que también se ha demostrado en otros estudios (Ramírez et al., 2017, Rosales et al., 2017b).

De la misma manera, algunos autores han detectado que las estructuras fúngicas dependen de la disponibilidad de nutrientes en el suelo, debido a que la simbiosis micorrízica está controlada por el suministro de carbono del hospedero, de modo tal que las estructuras fúngicas se pueden incrementar con un suministro adecuado de nutrientes; o, por el contrario, puede reducirse cuando se aplican cantidades de fertilizantes que exceden los requerimientos de los cultivos, ya que la entrega de los recursos del suelo a la planta hospedante a través de los hongos micorrízicos pierde importancia (Grman y Robinson, 2013; Castillo et al., 2014; Ramírez et al., 2017).

Un estudio desarrollado en Cuba sobre el efecto de la inoculación micorrízica en la rehabilitación de un cultivo de pasto Saboya corrobora su impacto beneficioso en el incremento de la altura de las plantas, en la mejora del porcentaje del área cubierta por dicho pasto, en la mejora del estado nutricional de las plantas, así como en el aumento del rendimiento de la biomasa, resultados que se atribuyen a la eficiencia de la cepa introducida Glomus cubense (Rosales et al., 2017a). 
Numerosos autores señalan que la aplicación de cepas eficientes de hongos micorrízicos arbusculares incrementa la efectividad de la absorción de los nutrientes tanto del suelo como de los fertilizantes, y como consecuencia se produce un incremento de la producción de biomasa del pasto (González, 2015; Rosales et al., 2017b; Canchani et al., 2018).

\section{CONCLUSIONES}

La inoculación de hongos micorrízicos arbusculares conjuntamente con la fertilización nitrogenada sobre el pasto Saboya constituye una opción válida para optimizar la absorción de minerales y nutrientes de este género mejorando su rendimiento y calidad, lo cual favorece el perfil alimentario del ganado en el cantón Chone y el país. De igual manera, constituye una práctica sustentable al disminuir el riesgo de contaminación ambiental y representa un ahorro económico dado el menor gasto en fertilizante.

El desafío más importante para el Ecuador, en este sentido, es el fomento de líneas investigativas dirigidas a la implementación de ambos métodos de fertilización para alcanzar elevados índices de productividad del pasto Saboya, tomando en cuenta factores como el tipo de suelo, la selección de cepas eficientes de micorrizas arbusculares y el manejo de cantidades de fertilizante nitrogenado acorde a las necesidades reales del suelo.

\section{LITERATURA CITADA}

Abbott, G. 1994. An ecological view of the fomation of VA mycorrhizas. Plant and Soil, 159(1): 69-78.

Aguilar, W., Arce, P., Galiano, F., \& Torres, T. 2015. Aislamiento de esporas y evaluación de métodos de inoculación en la producción de micorrizas en cultivos trampa. Tecnología en Marcha, Edición Especial: 5-14.

Alarcón, A., \& Ferrera-Cerrato, R. 2000. Biofertilizantes: importancia y utilización en la agricultura. Agricultura Técnica en México, 26(2): 191-203.

Álvarez, G., Vargas, J., Franco, F., Álvarez, P., Samaniego, M., Moreno, P., Chacón, E., García, A., Arana, R., Ramírez, J. 2016a. Rendimiento y calidad del pasto Megathyrsus maximys fertilizado con residuos líquidos de cerdo. Revista Electrónica de Veterinaria, 17(6): 1-9.

Álvarez, G., Vivas, R., Suárez, G., Cabezas, R., Jacho, T., Llerena, T, Valverde, H., Moreira, E., García, A., Chacón, M., Verdecia, D. 2016b. Componentes del rendimiento y composición química de Megathyrsus maximus en asociación con leguminosas. Revista Electrónica de Veterinaria, 17(12): 1-12.

Arnau, L., \& Sala, J. 2020. La revisión de la literatura científica: Pautas, procedimientos y criterios de calidad. Barcelona: Departament de Teories de 1'Educació i Pedagogia Social, Universitat Autónoma de Barcelona.

Astudillo, H. 2014. Determinación de la edad y la hora de corte sobre la concentración de carbohidratos solubles en el Panicum maximum (Pasto Guinea). Riobamba, Ecuador: Escuela Superior Politécnica de Chimborazo. Tesis de grado.

Barrera, J., \& Arango, G. 2015. Uso y Manejo de las Micorrizas: Investigación en cultivos. Universidad Nacional de Palmira. https://www.engormix.com/agricultura/articulos/uso -manejo-micorrizas-investigacion-t32322.htm

Barrera, S. 2009. El uso de hongos micorrízicos arbusculares como una alternativa para la agricultura. Facultad de Ciencias Agropecuarias, 7(1): 123-132.

Bernal, G., \& Moralez, R. 2006. Micorrizas: Importancia, Producción e Investigación en el Ecuador. Quito: ANCUPA.

Bernal, J. 2003. Pastos y forrajes tropicales. Producción y Manejo. Colombia: Editorial Ideagro 4ta Edición.

Bernal, J., \& Espinosa, J. 2003. Manual de nutrición y fertilización de pastos. Ecuador: International Plant Nutrition Institute-INPOFOS. https://www.academia.edu/6676325/MANUAL_DE _NUTRICION_Y_FERTILIZACION_DE_PASTO $\mathrm{S}$

Blanco, F., \& Salas, E. 1997. Micorrrizas en la agricultura: Contexto mundial e investigación realizada en Costa Rica. Agronomía Costarricense, 21(1): 55-67.

Blaxter, K., Wainman, F., Dewey, P., Davison, D., \& Gunn, J. 1971. The effects of nitrogenous fertilizer on the nutritive value of artificially dried grass. Journal of Agriculture Science, 76(1): 307-319.

Bolívar, A., Toro, M., Sandoval, M., \& López, M. 2009. Importancia ambiental y socioeconómica de las micorrizas en el cultivo de cacao. Caso: Hacienda Cata, Municipio Ocumare Costa de Oro, Estado Aragua, Venezuela. Agronomía Tropical, 59(4): 1-9. 
Cabalceta, G. 1999. Fertiliación y nutrición de Forrajes de altura. X1 Congreso Nacional Agronómico I J1/Congreso Nacional de Suelos, 3(54): 239-254.

Cáceres, O., Santana, H., \& Delgado, R. 1989. Influencia de la fertilización nitrogenada sobre el valor nutritivo $\mathrm{y}$ rendimiento de nutrimentos. Pastos y Forrajes, 12(2): 189-195.

Calderón, M., \& González, P. 2007. Respuesta del pasto Guinea (Panicun maximun cv. Likoni) cultivado en suelo ferralítico rojo lixiviado a la inoculación de hongos micorrízicos arbusculares. Cultivos Tropicales, 28(3): 33-37.

Canchani, A., Espaillat, R., \& López, J. 2018. El efecto y la aportación de la micorriza en el desarrollo de cultivos agrícolas. Revista Perspectiva en asuntos ambientales, 6(1): 12- 24.

Cano, M. 2011. Interacción de microorganismos benéficos en plantas: Micorrizas, trichoderma spp. y Pseudomonas spp. Una revisión. Revista U.D.CA Actualidad y Divulgación Científica, 14(2): 15-31.

Cárdenas, D., Garrido, M., Roncallo, B., \& Bonilla, R. 2014. Inoculación con Azospirullum spp y Enterobacter aglomerans en Pasto Guinea (Panicum maximum Jacq.) en el Departamento de Cesar Colombia . Revista Facultad Nacional de Agronomía- Medellín, 67(2): 7271-7280.

Cardona, G., Peña- Venegas, C., \& Arcos, A. 2008. Ocurrencia de hongos formadores de micorriza arbuscular asociados a ají (capsicum sp.) en la Amazonía colombiana. Agronomía Colombiana, 26(3): 459-470.

Castillo, C., Fredericksen, C., Koch, R., \& Sieverding, E. 2014. Effect of seed treatment with natural products onearly arbuscular mycorrhizal colonization of wheat by Claroideo glomus claroideum. J. Appl. Bot. Food Qual, 87: 117-123.

Castro, I. 2009. Análisis de la estructura y diversidad de las comunidades de hongos formadores de micorrizas asociados a plantas de interés ecológico en ambientes mediterráneos. España: Universidad de Granada. Tesis doctoral.

Castro, R., Mojica, R., León, J., Pabón, M., Carulla, J., \& Cárdenas, E. 2009. Balance de nitrógeno en pastura de gramíneas y pasturas de gramínea más Lotus uliginosus en la sabana de Bogotá, Colombia. Revista Corpoica- Ciencia y Tecnología agropecuaria, 10(1): 91-101.
Ceballos, M. 2019. Respuesta del pasto Saboya (Panicum maximun), establecido bajo dos sistemas de manejo a niveles de fertilización química en la zona de Babahoyo. Los Ríos- Babahoyo: Universidad Técnia de Babahoyo. Tesis previa obtención del título de Ingeniero Agropecuario.

Cerdas, R., \& Vallejos, E. 2011. Disponibilidad de biomasa del pasto guinea (Megathyrsus maximus) Tanzania con varias fuentes y dosis de nitrógeno en Guanacaste, Costa Rica. InterSedes, 12(23): 30-40.

Corredor, G., Otalvaro, D., \& Álbarez, C. 2003. Metodologías empleadas para el estudio de micorrrizas arbusculares. Bogotá: Programa Nacional de Recursos Biofísicos, CORPOICA.

Cuenca, G., Cáceres, A., Oirdobro, G., Hasmy, Z., \& Urdaneta, C. 2007. Las micorrizas arbusculares como una alternativa de agricultura sustentable en áreas tropicales. Interciencia, 1(2): 23-29.

Cunalata, Y. 2019. Caracterización morfológica de pasto Saboya (Panicum maximum Jacq.) en el cantón Babahoyo. Los Ríos, Babahoyo: Universidad Técnica de Babahoyo. Tesis de grado previa la obtención del título de Ingeniera Agrónoma.

Evans, D., \& Miller, M. 1990). The role of external mycelial network in the effect of soil disturbance upon vesicular-arbuscular mycorrhizal colonization in maize. New Phytol, 114(1): 65-71.

França, A., Borjas, A., Oliveira, E., Soares, T., Miyagi, E., \& Sousa, V. 2007. Parâmetros nutricionais do capimtanzânia sob doses crescentes de nitrogênio em diferentes idades de corte. Ciênc. Anim. Bras, 8(1): 695-703.

Garzón, L. 2016. Importancia de las micorrizas arbusculares (MA) para un uso sostenible del suelo en la Amazonía colombiana. Revista Luna Azul, 42(1): 217-234.

González, P. 2015. Manejo efectivo de la simbiosis micorrízica arbuscular via inoculación y la fertilización mineral en pastos del género Brachiaria. Habana: Editorial Universitaria.

González, P., Ramírez, J., Morgan, O., Rivera, R., \& Plana, R. 2015. Contribución de las inoculación micorrízica arbuscular a la reducción de la fertilización fosfórica en Brachiaria decumbens. Cultivos Tropicales 36(1): 135-142.

Grman, E., \& Robinson, T. 2013. Resource availability and imbalance affect plant-mycorrhizal interactions: 
afield test of three hypotheses . Ecology 94(1): 6271.

Guerra, B. 2008. Micorriza arbuscular. Recurso microbiológico en la agricultura sostenible. Tecnología en Marcha, 21(1): 191-201.

Guirao-Goris, J., Olmedo, A., \& Ferrer, E. 2008. El artículo de revisión. Revista Iberoamericana de Enfermería Comunitaria, 1(1): 1/16. http://revista.enfermeriacomunitaria.org/articuloCo mpleto.php?ID=7

Gutiérrez, M. 1996. Pastos y forrajes en Guatemala: su manejo y utilización, base de la producción animal. Guatemala: Facultad de Medicina Veterinaria y Zootecnia, Escuela de Zootecnia. Universidad de San Carlos de Guatemala.

Habte, M., \& Osorio, N. 2001. Arbuscular mycorrhizas: producing and applying arbuscular mycorrhizal inoculum. Hawaii: Collage of tropical agriculture and human resources. University of Hawaii at Manoa.

Jiménez, V. 2009. Efecto de diferentes inóculos comerciales de hongo micorrízico arbusculares sobre "Cedreka mantana" en etapa de vivero. Bogotá, Colombia: Universidad Distrital Francisco José de Caldas. Tesis de grado por el título de Ingeniera Forestal.

Kurle, J., \& Pfleger, F. 1994. The effects of the cultural practices and pesticides on VAM fungi in Micorrhizae and plant healt. Minesota: Editorial F. L. Pfleger and R.G.Lin- derman Minnesota APS Press.

León, R. 2003. Pastos y Forrajes, Producción y Manejo. Ecuador: Ediciones científicas Agustín Álvarez Cía Ltda.

Loayza, J. 2008. Evalución del pasto Saboya (Panicum maximum jacq) en el período de mínima precipitación, sometido a tres sistemas de pastoreo, en el acabado de toretes y vaconas Charbray, en la Hacienda San Antonio. Santo Domingo, Ecuador: Escuela Politécnica del Ejército.

López, V. 2015. Efecto de la fertilización de mantenimiento en el segundo año de establecida una pastura sobre su dinámica poblacional y algunas características del suelo en el CADET, Pichincha. Quito: Universidad Central del Ecuador. Tesis de grado previa a la obtención del título de Ingeniero Agrónomo.
Louis, I., \& Lim, J. 1988. Differential response and growth and mycorrhizal colonization on soybean to inoculation with two isolatess of Glomus c/aram in soils of diferent $P$ availability. Plant and Soil, 112(1): 37-43.

Lozano, J., Armbrecht, I., \& Montoya, J. 2015. Hongos formadores de micorrizas arbusculares y su efecto sobre la estructura de los suelos en fincas con manejos agroecológicos e intensivos. Acta Agroecología y Sistemas de Uso del Suelo, 64(4): 289-296.

Macías, D., Vargas, P., Solórzano, M., Mendoza, F., \& Intriago, F. 2017. Evaluación Agroproductiva del pasto Panicum maximun cv Mombaza en el Cantón el Carmen, Manabí -Ecuador. Revista ESPAMCIENCIA, 10(2): 78-84.

Maldonado, S. 2008. Estudio del comportamiento de las asociaciones micorrízicas en el material gemoplásmico de palma aceitera en Ecuador. Quito: Pontificia Universidad Católica del Ecuador. Tesis previa a la obtención del título de Licenciada en Ciencias Biológicas.

Memoria Técnica Cantón Chone. 2013. Proyecto Generación de Geoinformación para la Gestión del territorio a nivel nacional escala 1: 25000 Sistemas Productivos. Manabí: Memoria Técnica Cantón Chone.

Mendoza, R. 2002. Investigaciones sobre el uso del nitrógeno para la producción de pastos tropicales. Maracay- Venezuela: Instituto de Investigaciones Zootécnicas.

Mesquita, E., \& Neres, M. 2008. Morfogenese e composicao bromatológica de cultivares de Panicum maximum em funcao de adubação nitrogenada. Revista Brasileira de Saúde e Producion Animal, 9(1): 201-209.

Molina, M., Mahecha, L., \& Medina, M. 2005. Importancia del manejo de hongos micorrizógenos en el establecimiento de árboles en sistemas silvopastoriles. Revista Colombiana de Ciencias Pecuarias Universidad de Antioquía, 6(2):1-17.

Montaño, N., Camargo- Ricalde, S., García- Sánchez, R., \& Monroy, A. 2009. Micorrizas arbusculares en ecosistemas árido y semiáridos. México: Instituto Nacional de Ecología- SEMARNAT, Mundi-Prensa S.A. de CV, UAM- Iztapalapa, FES Zaragoza, UNAM. 
Moran, A. 2002. Composición química de pasto Panicum maximum común a diferentes niveles de fertilización nitrogenada. Esmeraldas- Ecuador: Universidad Luis Vargas Torres. Tesis por el título de Ingeniero Zootecnista.

Morán, C. 2019. Comparación de dos intervalos de Cortes del pasto Saboya (Panicum máximum jq) en su rendimiento de biomasa y valor nutritivo. Babahoyo, Ecuador: Universidad Técnica de Babahoyo. Tesis de grado previa a la obtención del título de Ingeniero Agrónomo.

Noda, Y. 2009. Las Micorrizas: Una alternativa de fertilización ecológica en los pastos. Pastos y forrajes, 32(2): 1-10.

Nuñez, J., Ñaupari, J., \& Flores, E. 2019. Comportamiento nutricional y perfil alimentario de la producción lechera en pastos cultivados (Panicum maximum Jacq). Revista Peruana de Investigación Veterinaria, 30(1): 178-192.

Núñez, M. 2018. Efecto de Azospirillum sp., Trichoderma harzianum y micorrizas en la producción de pasto Marandú (Brachiaria brizantha) y pasto Guinea (Panicum maximum). Zamorano, Honduras: Escuela Agrícola Panamericana. Proyecto especial de graduación presentado como requisito parcial para optar por el título de Ingeniera Agrónoma en el Grado Académico de Licenciatura.

Parodi, G., \& Pezzani, F. 2011. Micorrizas arbusculares en dos gramíneas nativas de Uruguay en áreas con y sin pastoreo. Agrociencia Uruguay, 15(2): 1-10.

Peñaherrera, A. 2015. Producción y calidad forrajera de pasto Saboya (Panicum maximum Jacq) a diferentes edades y alturas de corte. Santo Domingo de los Tsáchilas: Universidad de las Fuerzas Armadas (ESPE). Tesis de grado previa a la obtención del título de Ingeniero Agropecuario.

Peoples, M., Herridge, D., \& Ladha, J. 1994. Management of biological nitrogen fixation for the development of more productive and sustainable agricultural systems. Plant and Soil. Symposium on biological nitrogen fixation for sustainable agriculture at the 15 th congress of soil science. Acapulco, México, 174(1): 3-28.

Pérez, A., Rojas, J., \& Montes, D. 2011. Hongos formadores de micorrizas arbusculares: una alternativa biológica para la sostenibilidad de los agrosistemas de praderas en el Caribe colombiano. Revista Colombiana Ciencia Animal, 3(2): 366-385.
Pérez, O. 2014. Eficiencia de uso de nitrógeno en pasturas de Panicum maximun y Brachiaria sp. solas y asociadas con Pueraria phaseoloides en la altillanura colombiana. Bógota: Universidad Nacional de Colombia. Tesis de opción al grado de Maestría en Producción Animal.

Ramírez, J., González, P., Salazar, X., LLanes, D., Rivera, R., Hernández, A., \& Plana, R. 2017. Inoculación micorrízico arbuscular y reducción de la fertilización orgánica y nitrogenada en Megathyrsus maximus cv. Liconi. Pastos y Forrajes, 40(2): 1-9.

Ramírez, J., Herrera, R., Leonard, I., Cisneros, M., Verdecia, D., \& Álvarez, Y. 2012. Rendimiento de indicadores de calidad en Panicum maximum vc. Likon en el Valle del Cauto, Cuba. Revista Electrónica Veterinaria, 13(4): 1-8.

Reynolds, S. 1982. Contributions to yield, nitrogen fixation and transfer by local and exotic legumes in tropical grass-legume mixtures in Western Samoa. Tropical Grasslands, 16(2): 76-80.

Riera, M., \& Medina, N. 2005. Influencia de las micorrizas sobre las poblaciones bacterianas y su efecto sobre los rendimientos en secuencias de cultivos. Cultivos Tropicales, 26(4): 21-27.

Rosales, P., González, P., Ramírez, J., \& Arzola, J. 2017a. Efecto de la inoculación micorrízica arbuscular en la rehabilitación de un pastizal cultivado de guinea (Megathyrsus maximus cv. Likoni). Revista Cultivos Tropicales, 38(4): 1-25.

Rosales, P., González, P., Ramírez, J., \& Arzola, J. 2017 b. Selección de cepas eficientes de hongos micorrízicos arbusculares para el pasto Guinea (Megathyrsus maximus cv. Likoni). Cultivos tropicales, 38(1): 2430 .

Santos, I., Pinto, J., Siqueira, J., Morais, A., \& Santos, C. 2002. Influencia do fósforo, micorriza e nitrogenio no conteúdo de minerais de Brachiaria brizantha e Arachis pintoj consorciados. Revista Brasileña de Zootecnia, 31(1): 605-616.

Seguel, A. 2014. El potencial de las micorrizas arbusculares en la agricultura desarrollada en zonas áridas y semiáridas. IDESIA, 32(1): 3-8.

Segura, N. 2007. Evaluación del Pasto Saboya (panicum maximum) sometido a tres sistemas de manejo, en el acabado de toretes y vaconas Charbray en la Hacienda San Antonio. Santo Domingo Ecuador: Escuela Politécnica del Ejercito. Tesis de grado 
previa a la obtención del título de Ingeniero Agrónomo.

Sieverding, E. 1991. Vesicular-arbuscular mycorrhizal management in tropical agrosystems. Federal Republic of Germany: Technical Cooperation.

Usuga, C., Castañeda, D., Franco, A., Gómez, F., \& Lopera, C. 2008. Efecto de la micorrización y la fertilización en la acumulación de biomasa en plantas de banano( Musa AAA cv. Gran Enano) (Musaceae). Revista Facultad Nacional Agronomía- Medellín, 61(1): 4269-4278.

Valencia, C., \& Zúñiga, D. 2015. Análisis de la presencia natural de micorrizas en cultivos de algodón (Gossypium barbadense L.) inoculados con Bacillus megaterium y/o Bradyrhizobium yuanmingense. Ecología Aplicada, 14(1): 65-69.
Vargas, J., Leonard, I., Uvidia, H., Ramírez, J., Torres, V., Andino, M., \& Benítez, B. 2014. El crecimiento del pasto Panicum maximum cv Mombaza en la Amazonía Ecuatoriana. Revista Electrónica de Veterinaria (REDVET), 15(9): 1-7.

Vélez, S. 2009. Evaluación del pasto Saboya (Panicum maximum Jacq) in vitro y corte sometido a diferentes dosis de fertilización nitrogenada. Calceta, Ecuador: Escuela Superior Politécnica Agropecuaria de Manabí Manuel Félix López.

Verdecia, D., Ramírez, J., Leonard, I., Pascual, Y., \& López, Y. 2008. Rendimiento y componentes del valor nutritivo del Panicum maximum cv. Tanzania. Revista Electrónica de Veterinaria, 9(5): 1-9.

Whitehead, D. 1995. Grassland Nitrogen. Gran Bretaña: Ediciones CAB International, Guildford. 